\title{
Risque radiologique et faibles doses : entre faux débat et expérience
}

\author{
J. LOCHARD*
}

(Manuscrit reçu le 11 février 1994)

RÉSUMÉ L'information des travailleurs et du public sur les rayonnements ionisants et leurs effets potentiels est très superficielle. La communauté scientifique comme les experts qui établissent les normes de base de radioprotection n'ont pas vraiment réussi à faire passer un message clair et constructif sur les principes qui fondent l'évaluation et la gestion du risque radiologique. Le débat sur les faibles doses est, à cet égard, révélateur du déficit de connaissances dans ce domaine. Des efforts en matière d'éducation ainsi que l'“expérience directe" de la radioactivité dans tous les domaines devraient, à terme, favoriser l'émergence d'une véritable culture du risque radiologique apte à réconcilier le public avec les techniques et les hommes qui les mettent en ouvre et à restaurer la confiance dans les institutions qui sont en charge de l'évaluation et de la gestion du risque radiologique.

ABSTRACT The information of the workers and the public on ionizing radiation and their potential effects is very superficial. The scientific community, as well as the experts in charge of establishing basic radiation protection standards, have never really succeeded to transmit a clear and constructive message on the fundamental principles underlying the assessment and management of radiological risk. The on-going debate on low doses is a good illustration of the deficit in knowledge in this field. An educational effort, with "direct experience" of radioactivity in all domains, should, in the future, facilitate the emergence of a true radiological risk culture. This should help both in reconciling the public with the techniques and the people involved and restoring the trust in the institutions in charge of the evaluation and the management of radiological risk.

\section{Introduction}

Les connaissances du public, comme celles de la plupart des travailleurs exposés aux rayonnements ionisants, concernant les niveaux d'exposition aux différentes catégories d'irradiation auxquels ils sont soumis (naturelle, médicale, industrielle), ou encore les effets potentiels de ces rayonnements sur la santé, sont très superficielles. Ces sujets sont absents de l'enseignement de base à l'école et, par ailleurs, ils ne sont presque jamais abordés par les médias. Leur traitement n'est, en fait, assuré que de façon incidente à l'occasion d'événements accidentels plus ou moins graves, évidemment peu propices à une démarche pédagogique. Il résulte de cette situation que pratiquement n'im-

* Centre d'étude sur l'évaluation de la protection dans le domaine nucléaire (CEPN), BP 48, 92263 Fontenay-aux-Roses Cedex. 
porte quel discours peut être tenu sur le risque radiologique sans qu'aucune sanction intellectuelle ne vienne y mettre un terme. Le grand public est prêt à écouter tout argument, mais aussi à l'adopter, dès lors que celui qui le soutient sait être convaincant.

Le débat sur les effets sanitaires potentiels associés à l'exposition aux faibles doses de rayonnement est, à cet égard, caractéristique de cette absence d'esprit critique. Depuis maintenant plusieurs décennies, partisans et opposants au développement de l'énergie nucléaire s'affrontent sur cette question avec les mêmes sempiternels arguments sans tenir compte de l'état actuel des connaissances et des pratiques en matière d'évaluation et de gestion du risque radiologique. Force est de constater que la communauté scientifique, qui participe au développement des connaissances dans le domaine des effets des rayonnements, de même que les experts qui établissent les normes de base de la radioprotection, n'ont pas vraiment su, jusqu'ici, apporter les explications susceptibles de dépassionner ce débat et, dans une perspective plus globale, de développer une attitude rationnelle et responsable vis-à-vis du risque radiologique.

Finalement, la situation actuelle est assez paradoxale. En effet, les efforts de protection réalisés à ce jour ont permis de réduire les niveaux de risque résiduels auxquels sont soumis le public et les travailleurs, en particulier dans le domaine du cycle électronucléaire, à des niveaux extrêmement faibles qui n'ont pas lieu de soulever une inquiétude quelconque dans la population. Néanmoins, le déficit en matière de connaissances fait le jeu des discours alarmistes qui prétendent que ces niveaux d'exposition sont malgré tout dangereux, cherchant ainsi à utiliser l'argument du risque pour faire passer des messages dont la finalité sanitaire n'est pas, en réalité, prioritaire. A l'opposé, ce même déficit favorise l'éclosion de discours simplistes et faussement rassurants sur l'absence de risque aux faibles doses qui génèrent une suspicion latente à l'égard des scientifiques, des experts et des responsables d'installations et qui les placent en situation d'accusés dès lors qu'un événement survient. Il est évident qu'un tel contexte n'est propice ni à un dialogue constructif ni à la transparence en matière de communication.

Après un rappel des arguments qui alimentent le débat sur les faibles doses et des principes qui fondent l'évaluation et la gestion du risque radiologique, cet article propose quelques éléments de réflexion qui pourraient favoriser l'émergence d'une réelle acceptation des risques associés à l'utilisation des rayonnements ionisants.

\section{Le débat sur les faibles doses}

Le débat sur les effets potentiels sur la santé associés à l'exposition aux faibles doses de rayonnement est caractéristique des conséquences du déficit des connaissances en matière de risque radiologique. De façon très schématique, deux thèses antagoniques s'opposent dans ce débat. 
Pour les uns, le postulat de base est celui de l'existence d'un seuil d'exposition en dessous duquel les rayonnements ionisants n'ont aucun effet décelable sur la santé. Comme principal argument, les défenseurs de cette thèse mettent en avant le fait que les nombreuses études réalisées à ce jour sur les effets potentiels des faibles doses n'ont jamais permis de démontrer de façon irréfutable l'existence de tels effets. Même si ce constat ne saurait constituer une preuve en soi, il renforce néanmoins leur intime conviction qu'un seuil doit exister. Quant à la démonstration de l'existence de ce dernier, ce n'est qu'une affaire de moyens et de temps et les progrès de la science devraient finir par leur donner raison. Cette thèse est partagée par un certain nombre de responsables de l'industrie électronucléaire, tant au niveau des autorités compétentes que des exploitants, mais surtout par le milieu médical. La confrontation quotidienne à la radioactivité de nombreux médecins dans l'exercice de leur pratique est probablement un facteur explicatif de leur attitude. Pour les partisans du seuil, les normes de protection qui ont été progressivement élaborées sur la base des travaux du Comité scientifique des Nations-Unies sur les effets des rayonnements (UNSCEAR) et des recommandations de la Commission internationale de protection radiologique (CIPR) $[9,2]$ sont suffisamment restrictives pour garantir l'innocuité des niveaux d'exposition tels qu'ils résultent des pratiques actuelles, et il n'y a donc pas de raisons pour accorder aux faibles doses un intérêt particulier.

Le postulat qui fonde l'autre logique est que les rayonnements sont dangereux, quel que soit le niveau d'exposition, et qu'il convient donc de réduire les expositions le plus bas possible, l'idéal étant de les supprimer complètement. L'argument le plus souvent avancé pour justifier cette position consiste à affirmer que le risque associé aux faibles doses est sous-estimé. S'appuyant sur des travaux de scientifiques dits "indépendants", qui remettent en cause les conclusions auxquelles a abouti la communauté des experts internationaux spécialisés dans le domaine de la radioprotection, les tenants du "risque zéro" militent donc pour une réduction des normes. Le crédit accordé à ces travaux "indépendants", plutôt qu'aux conclusions sur la base desquelles un consensus a pu s'établir sur le plan international en la matière, se justifie essentiellement par le fait qu'il est jugé difficile de faire confiance aux experts compte tenu de leurs liens privilégiés avec "l'establishment" nucléaire [8]. Le recours à la suspicion comme élément de démonstration n'est évidemment pas très convaincant. Il place, cependant, les experts dans une position délicate puisque ces derniers sont actuellement dans l'incapacité de démontrer scientifiquement l'existence d'un seuil pour les effets stochastiques. Les détracteurs de l'industrie électronucléaire partagent, pour la plupart, cette seconde thèse et ils trouvent dans cette incapacité à démontrer l'existence d'un seuil pour les faibles doses un argument qui peut paraître persuasif à première vue.

Un autre point de focalisation du débat est la relation exposition-risque (ou dose-effet) sur la base de laquelle est exprimé quantitativement le risque associé aux faibles doses. Pour les tenants de l'innocuité de ces dernières, la relation dose-effet n'est qu'une construction intellectuelle qui n'a aucun fondement 
scientifique sérieux. Elle est établie sur la base d'un ensemble d'hypothèses qui ne sauraient objectiver l'existence d'effets aux faibles doses. Pour les partisans de la logique selon laquelle le risque existe quel que soit le niveau d'exposition, la relation dose-effet est, par contre, considérée comme un élément de preuve et le problème n'est pas tant celui de la validité scientifique de la relation, qui n'est pas remise en cause, mais celui de sa "justesse" quant à l'estimation du risque. Les uns comme les autres semblent donc oublier que la relation doseeffet n'est que le résultat d'un consensus d'experts à un moment donné susceptible d'être remis en cause à tout moment en fonction de l'évolution des connaissances. Le statut de la relation dose-effet, à mi-chemin entre les connaissances scientifiques et les jugements de valeurs [6], permet donc aux uns de la réfuter au nom de la science et aux autres de la critiquer pour insuffisance, tout en l'utilisant implicitement pour justifier l'existence d'effets aux faibles doses. Dans ces conditions, on peut comprendre que le dialogue soit difficile, d'autant plus que chacun des discours n'est pas exempt d'une certaine ambiguité, voire d'une certaine mauvaise foi. D'un côté, en effet, on s'appuie sur le fait qu'on n'a jamais pu mettre en évidence scientifiquement l'existence d'effets aux faibles doses, mais on oublie qu'on n'a jamais pu démontrer non plus leur non existence. De l'autre, on s'appuie directement sur la relation dose-effet, en oubliant que cette dernière ne traduit pas la réalité, mais seulement un ensemble d'hypothèses avancées pour fonder l'action en matière de radioprotection.

Il est intéressant, par ailleurs, de souligner que la logique développée par les opposants de l'industrie électronucléaire en matière d'effets des faibles doses place les défenseurs de la thèse de l'existence du seuil en situation de devoir en permanence se justifier. Sur un plan stratégique, elle vise aussi à faire pression de façon permanente pour un renforcement des normes de radioprotection et, en particulier, pour la réduction des limites de dose, ce qui est perçu par l'industrie comme une source de contraintes de gestion et donc de coûts supplémentaires. On peut comprendre dans un tel contexte l'enjeu que constitue la question de la révision des limites de dose puisque toute baisse de ces dernières pourrait apparaître aux yeux de l'opinion comme une confirmation de la justesse de la position des opposants et un désaveu pour les responsables de l'électronucléaire. Un tel désaveu serait d'autant plus mal perçu qu'il aurait pour conséquence d'une part de remettre en question la crédibilité scientifique des experts chargés d'étudier les effets des rayonnements et de fixer des normes adaptées et, d'autre part, de faire émerger un doute dans l'esprit des personnes, en particulier les travailleurs, et qui, ayant été exposées à des niveaux proches des anciennes normes, pourraient estimer ne pas avoir été correctement protégées jusque là.

Le débat sur les faibles doses apparaît donc comme un "vrai faux débat". La confrontation des deux thèses, apparemment inconciliables, tient pour une grande part à un réel manque de connaissances quant aux fondements de la gestion du risque radiologique. En particulier, la confusion qui perdure dans les esprits entre les modalités de gestion du risque associé aux effets déterministes 
(du type érythèmes, brûlures, nécroses...) et celles qui concernent les effets stochastiques (cancers et effets héréditaires) est à l'origine de la plupart des contresens qui sous-tendent le débat.

\section{La gestion des effets stochastiques}

Comparativement à la gestion des effets déterministes qui repose sur le modèle classique du seuil, découlant directement des connaissances scientifiques sur les modalités d'apparition des effets et permettant d'assurer une garantie individuelle de non apparition de ces effets, la gestion des effets stochastiques apparaît comme beaucoup plus complexe. Devant l'impossibilité de démontrer scientifiquement l'existence d'un seuil aux faibles doses pour les effets stochastiques, et afin de "minimiser le regret" au cas où le doute serait un jour levé, la communauté internationale des experts, essentiellement sous l'égide de la CIPR, a adopté, dès les années cinquante, une attitude prudente du point de vue de l'action qui consiste à faire comme si un tel seuil n'existait pas [3]. La gestion des effets stochastiques repose donc sur le principe de précaution que l'on peut qualifier de principe éthique dans la mesure où il ne renvoie pas directement à la connaissance scientifique mais à un choix raisonné en vue de l'action [5]. De ce choix découle logiquement la volonté de réduire le plus bas possible les niveaux d'exposition tout en tenant compte, d'une part, que les ressources de protection ne sont pas illimitées et que, d'autre part, la réduction des risques pour certains groupes peut induire des transferts de risques vers d'autres groupes dès lors que les niveaux résiduels sont faibles. Cette démarche plus connue sous le nom de principe d'optimisation (également appelé principe ALARA) constitue le pilier central du système de protection en matière de faibles doses qui conduit à maintenir les expositions aussi bas qu'il est raisonnablement possible, compte tenu des facteurs économiques et sociaux, en dessous des limites [7]. Dans ce contexte, la limite n'a plus du tout la signification d'un seuil en dessous duquel aucun effet ne devrait apparaître, comme dans le cas des effets déterministes, mais elle est la garantie donnée à chaque individu que le risque résiduel individuel qui lui est associé est jugé tolérable du point de vue social, comparativement aux autres risques auxquels il est soumis soit en tant que travailleur soit en tant que membre du public.

On peut concevoir aisément que sur la base d'un tel édifice la communication en matière de risque radiologique soit assez difficile et qu'elle constitue même un réel défi. De nombreux experts considèrent que le domaine est trop complexe pour pouvoir être compris par tout le monde et qu'en conséquence il faut s'en tenir à un message simple et rassurant. Cette approche conduit le plus souvent à réduire la gestion du risque radiologique à un problème de respect de limite, vidant ainsi de sa substance la problématique du risque résiduel.

L'objectif devrait être, au contraire, de rendre compréhensible pour le plus grand nombre les grands principes qui fondent cette gestion, à savoir : 
l'absence de certitudes scientifiques quant à l'existence d'un seuil d'apparition pour les effets stochastiques, la prudence qui conduit à adopter une relation dose-effet linéaire sans seuil, la responsabilité qui conduit à rechercher des niveaux de protection compatibles avec les ressources sociales disponibles et les transferts de risques entre groupes de population et, enfin, la transparence des processus décisionnels en matière de choix de protection. Le point le plus important est certainement de bien montrer, exemples à l'appui, que le risque nul n'est pas tenable ni sur le plan de l'allocation sociale des ressources ni sur celui de l'équité. La protection contre le risque radiologique ne peut pas se faire à n'importe quel prix dès lors que chaque individu est soumis à un ensemble de risques résiduels et que les ressources que peut consacrer la collectivité à la protection de ses membres ne sont pas illimitées. De même, la protection d'un groupe de population ne peut pas se faire au détriment d'un autre groupe dès lors qu'il existe de fortes interactions en matière de transferts de risques entre ces groupes. On oublie trop souvent, par exemple, que la protection du public peut induire des risques supplémentaires pour les travailleurs et que vouloir supprimer un risque pour les générations actuelles peut se traduire par un transfert de ce risque vers les générations futures.

\section{4. Éducation et expérience}

La clarification de la démarche qui fonde la gestion du risque radiologique devrait être fondée sur deux volets.

Le premier de ces volets, à finalité didactique, centré sur les concepts et les principes fondamentaux de la radioprotection, aurait pour objectif d'améliorer la compréhension de tout un chacun sur les rayonnements et sur leurs effets sanitaires ainsi que sur la philosophie qui guide la protection. A l'attention de tous ceux qui participent de près ou de loin à l'évaluation et à la gestion du risque radiologique, il conviendrait essentiellement de repositionner le débat sur les faibles doses sur des bases correctes du point de vue scientifique et éthique. En ce qui concerne le public au sens large, l'objectif devrait être l'amélioration du niveau de connaissances, d'une part à travers l'éducation à l'école et d'autre part à travers l'information relative à toutes les activités et les situations impliquant les rayonnements ionisants. Dans ce cadre, il conviendrait d'expliquer clairement la différence entre les rayonnements naturels et artificiels, les effets stochastiques et les effets déterministes, la prévention et la précaution, le principe de limitation et le principe d'optimisation, ainsi que le rôle respectif de la science et de l'éthique dans la gestion du risque radiologique. Par ce biais, il serait ainsi possible d'instaurer les bases d'un vrai débat social sur le risque résiduel en relation avec sa justification, c'est-à-dire le bénéfice social attendu des pratiques et des activités dont il découle. Un tel débat permettrait également de montrer que la démarche adoptée pour les rayonnements ionisants est pertinente et peut avoir valeur exemplaire pour la gestion de nombreux autres risques technologiques, industriels et sociétaux [4]. 
Concrètement, cette démarche d'explication pourrait s'appuyer sur un ensemble de moyens déjà existants qui, redynamisés, devraient être largement suffisants pour assurer un impact significatif : système éducatif, associations professionnelles de type Société française de radioprotection (SFRP) ou Société française des physiciens d'hôpital ((SFPH), conférences et publications, sans oublier les médias. Il conviendrait également d'adopter une attitude plus positive vis-à-vis des grands comités internationaux chargés de l'évaluation et de la gestion du risque radiologique (en particulier l'UNSCEAR et la CIPR), qu'il faudrait mieux faire connaître afin qu'ils apparaissent aux yeux de l'opinion, non pas comme les instruments de tel ou tel groupe de pression, mais comme de véritables comités de réflexion et d'éthique, ce qu'ils sont en réalité.

Le second volet devrait porter sur le développement d'actions pratiques pour familiariser le public avec la radioactivité. En effet, l'une des difficultés majeures en matière de communication et de compréhension dans le domaine des rayonnements ionisants réside dans leur caractère abstrait comme d'ailleurs celui du risque qu'ils représentent. Les rayonnements sont invisibles, inodores, inaudibles et indolores et, en dehors des accidents graves d'irradiation ou de contamination qui restent extrêmement rares, le public n'a pas la possibilité de les matérialiser concrètement et d' "expérimenter" le risque qui leur est associé. Aux côtés très abstraits des principes et des concepts qui fondent l'évaluation et la gestion du risque radiologique, vient donc s'ajouter l'impossibilité d'un "vécu" capable d'objectiver ce risque et de le mettre en perspective avec les autres risques de la vie quotidienne. Il paraît souhaitable de développer une réflexion à ce niveau en vue de construire une perception des rayonnements davantage fondée sur la réalité et l'expérience que sur des craintes mal fondées.

L'accent dans ce domaine devrait en priorité porter sur la mise en évidence concrète de l'existence de la radioactivité et sa mesure dans tous les domaines professionnels, environnementaux et médicaux. On peut envisager diverses initiatives relativement faciles à mettre en œuvre : dosimètres personnels, carnet d'exposition personnel, campagnes d'information sur le radon et sur les irradiations médicales, développements de laboratoires de mesures indépendants soumis à des règles déontologiques strictes (intercomparaison annuelle, entre autres, sous la responsabilité des autorités compétentes), association des universités scientifiques à la surveillance radiologique des sites et de l'environnement, mention de la radioactivité sur les étiquettes des produits de consommation, etc. Cette liste n'est évidemment pas exhaustive et d'autres actions devraient pouvoir contribuer à "matérialiser" les niveaux d'exposition et familiariser chacun avec la réalité incontournable des rayonnements.

\section{Conclusion}

La gestion du risque radiologique est un domaine de l'action humaine dans lequel la science n'est pas encore en mesure d'apporter une réponse définitive quant aux conséquences potentielles sur la santé des niveaux d'exposition aux- 
quels sont soumis le public, les travailleurs et les patients dans le cadre des diverses activités dans lesquelles les rayonnements ionisants sont impliqués. Les connaissances accumulées jusqu'à présent sont, cependant, suffisantes pour que cette limitation des connaissances ne soit pas de nature à remettre en cause les avantages retirés par la société de l'utilisation des propriétés de l'atome et des rayonnements ionisants [1]. En ce qui concerne la part connue des conséquences, l'infrastructure technique, organisationnelle et réglementaire permet d'apporter une garantie suffisante à chaque individu quant à sa sécurité. Cette garantie a évidemment un coût social et elle suppose de plus une vigilance de tous les instants de l'ensemble des acteurs concernés, comme dans la plupart des activités humaines qui comportent des risques. Quant à la part des conséquences encore mal connue sur le plan scientifique, c'est sur la base d'une attitude de prudence qu'ont été fixés les principes de protection. Dans ce cas, la garantie porte sur un niveau de risque résiduel considéré comme tolérable compte tenu du contexte économique et social. L'émergence de ces considérations économiques et sociales au cœur de la protection n'est que la reconnaissance explicite du souci de l'autre et de l'idée de justice et d'équité dans la recherche du risque résiduel acceptable.

La communication actuelle entre les experts et le public pèche par un excès de simplification, doublé chez certains responsables d'une tendance à minimiser les effets potentiels des rayonnements - ce qui, globalement, peut produire un résultat opposé à celui recherché. En effet, plutôt que d'informer et d'éduquer le public et les travailleurs sur le risque associé aux rayonnements ionisants et aux modalités de sa gestion, le discours dominant se caractérise, le plus souvent, par une sorte de plaidoyer pour essayer de convaincre, par le biais d'arguments simples et directs, que les irradiations associées aux pratiques médicales et industrielles courantes sont bien maîtrisées et ne posent pas de problèmes particuliers dès lors que les limites de dose réglementaires sont respectées. Bien que compréhensible et par bien des aspects légitime, cette approche, qui se veut à la fois rassurante sur le risque et exemplaire quant à son contrôle, place en situation très inconfortable ses défenseurs qui se retrouvent en position d'accusés dès lors qu'un incident ou une affaire, même banale, survient. Le doute s'insinue alors dans les esprits quant à la maîtrise réelle des situations et, en l'absence de repère pour relativiser les niveaux de risque mis en jeu, la critique peut se développer avec ses ambiguïtés sur les plans scientifique et éthique et ses arrières pensées sur le plan politique.

Il est important que les principes sur lesquels repose la protection radiologique deviennent progressivement compréhensibles dans leurs grandes lignes par tout le monde. C'est autant par l' expérience directe" des rayonnements que par la connaissance théorique de leurs effets et de leurs modalités de gestion qu'il sera possible de faire émerger une véritable culture du risque radiologique adaptée aux réalités économiques, sociales et culturelles actuelles, de réconcilier le public avec les techniques et les hommes qui les utilisent et de restaurer la confiance dans les institutions qui sont en charge de l'évaluation et de la gestion de ce risque. 


\section{RÉFÉRENCES}

[1] AGENCE POUR L'ENERGIE NUCLEAIRE (AEN) DE L'OCDE - La radioprotection au seuil du XXI siècle, compte rendu d'une réunion de travail de l'AEN, Paris, 11-13 janvier 1993. Paris : OCDE, 1993.

[2] COMMISSION INTERNATIONALE DE PROTECTION RADIOLOGIQUE (CIPR) Recommandations 1990... (Publication CIPR $n^{\circ}$ 60). Oxford : Pergamon Press, 1993.

[3] INTERNATIONAL COMMISSION ON RADIOLOGICAL PROTECTION (ICRP) Recommendations..., revised at the 6th International congress of radiology, London, 1950. Brit. J. Radiol., 1951, 24, 46-53.

[4] J.M. LEHN - La lumière du futur. Le Monde, 27 avril 1994.

[5] J. LOCHARD, M.-C. GRENERY-BOEHLER - Les bases éthiques et juridiques du principe d'optimisation de la radioprotection. Bull. Droit Nucl., 1993, $\mathrm{n}^{\circ}$ 52, 9-28.

[6] NATIONAL COUNCIL ON RADIATION PROTECTION AND MEASUREMENTS (NCRP) - Limitation of exposure to ionising radiation (NCRP Report $\left.n^{\circ} 116\right)$. Bethesda : NCRP, 1993.

[7] P.J. STOKELL, J.R. CROFT, J. LOCHARD, J. LOMBARD - ALARA : from theory towards practice. Report EUR-13796 EN. Bruxelles : Commission des Communautés Européennes, 1991.

[8] A.R. TAMPLIN, J.W. GOFMAN - Population control through nuclear pollution. Chicago : Nelson-Hall company, 1970.

[9] UNITED NATIONS SCIENTIFIC COMMITTEE ON THE EFFECTS OF ATOMIC RADIATION (UNSCEAR) - Sources and effects of ionizing radiation, 1993 report to the General assembly. New-York : United Nations, 1993. 